\title{
Detection of event-related potentials in individual subjects using support vector machines
}

\author{
Hossein Parvar • Lauren Sculthorpe-Petley • \\ Jason Satel • Rober Boshra • Ryan C. N. D'Arcy • \\ Thomas P. Trappenberg
}

Received: 28 May 2014/Accepted: 8 November 2014/Published online: 25 November 2014

(C) The Author(s) 2014. This article is published with open access at Springerlink.com

\begin{abstract}
Event-related potentials (ERPs) are tiny electrical brain responses in the human electroencephalogram that are typically not detectable until they are isolated by a process of signal averaging. Owing to the extremely small size of ERP components (ranging from less than $1 \mu \mathrm{V}$ to tens of $\mu \mathrm{V}$ ), compared to background brain rhythms, statistical analyses of ERPs are predominantly carried out in groups of subjects. This limitation is a barrier to the translation of ERP-based neuroscience to applications such as medical diagnostics. We show here that support vector machines (SVMs) are a useful method to detect ERP components in individual subjects with a small set of electrodes and a small number of trials for a mismatch negativity (MMN) ERP component. Such a reduced experiment setup is important for clinical applications. One hundred healthy individuals were presented with an auditory pattern containing pattern-violating deviants to evoke the MMN. Two-class SVMs were then trained to classify averaged ERP waveforms in response to the standard tone (tones that match the pattern) and deviant tone stimuli
\end{abstract}

H. Parvar · R. Boshra $\cdot$ T. P. Trappenberg $(\square)$

Faculty of Computer Science, Dalhousie University, 6050

University Avenue, P.O. Box 1500, Halifax, NS B3H 4R2,

Canada

e-mail: tt@cs.dal.ca

L. Sculthorpe-Petley

Biomedical Translational Imaging Centre (BIOTIC), IWK

Health Centre, Halifax, NS, Canada

J. Satel

School of Psychology, Faculty of Science, University of

Nottingham Malaysia Campus, Semenyih, Selangor, Malaysia

R. C. N. D'Arcy

Applied Sciences, Simon Fraser University, Surrey, BC, Canada (tones that violate the pattern). The influence of kernel type, number of epochs, electrode selection, and temporal window size in the averaged waveform were explored. When using all electrodes, averages of all available epochs, and a temporal window from 0 to $900-\mathrm{ms}$ post-stimulus, a linear SVM achieved $94.5 \%$ accuracy. Further analyses using SVMs trained with narrower, sliding temporal windows confirmed the sensitivity of the SVM to data in the latency range associated with the MMN.

Keywords Support vector machine .

Event-related potentials - Mismatch negativity .

Electroencephalography $\cdot$ Neuroscience $\cdot$ Diagnostic

\section{Introduction}

Event-related potential (ERP) components are specific deflections in the averaged waveform, which are considered to be tied to discrete stages of neural processing. They are extracted from trials or epochs of electroencephalogram (EEG) data that are temporally locked to a repeated event, either a stimulus or a behavioural response, which are segmented from the continuous EEG recording and then averaged together. This averaging process reduces the amplitude of brain activity that is unrelated to the event, thus presumed to be random with respect to it in time, and retains brain responses that are temporally related to the event (see [1] for a full description of ERP derivation). The most commonly used definition of an ERP component was outlined by Näätänen and Picton in 1987 [2]. Their definition emphasizes that an ERP component is described not only by the brain regions that contribute to its production, but also by the experimental parameters which must be satisfied in order to observe the response. ERPs can be 
evoked by stimulation in any sensory modality and can also occur in response to motoric and cognitive events.

In the auditory domain, ERPs can be used to index processing all the way from early sensory processing in the brainstem to cortical language processing. The auditory brainstem response (ABR) has a long history of use in both neurology and audiology [3]. Cortical ERPs have yet to become a routine part of clinical practice, even in audiology (reviewed in [4]) and cognitive assessment of higher functions like language [5-7] where their potential utility is very clear.

In large part, the failure of cortical ERPs to be translated into clinical practice is due to the requirements of the signal-averaging process. Many repetitions of the evoking stimulus or event must be presented in order to isolate ERPs, which can lead to long testing times. Another important consideration for the application of ERPs for both clinical and research purposes is the need to statistically verify the presence of the response. Given the low signal-to-noise ratio of these responses versus background EEG, the typical approach in ERP research is to perform univariate statistics across groups, or across experimental conditions, using amplitudes that are measured in the averaged waveform [8].

Many groups have proposed solutions to verify the presence of ERPs in individual subjects, and even single epochs, by applying techniques such as wavelet analysis [9-11], independent component analysis [12], integrated waveforms [13], and nonparametric analyses [14]. In some very specific clinical situations, the approach of repeating stimulation until a statistical threshold is reached [15], or using basic $\mathrm{t}$-scores to evaluate the presence or the absence of a particular component [16, 17], has been examined.

In particular, the field of Brain-Machine Interfaces (BMIs) has yielded much progress in the single-subject detection of responses like ERPs. This area seeks to use brain imaging data, mostly noninvasive technology, such as EEG, to identify brain waves in continuous EEG recordings that can be interpreted as commands by a computer [18]. The progress that has been seen in this area is mainly due to advances in machine learning techniques. While results in BMI research show that signals can be extracted from single trials, here we investigate specifically the use of machine learning techniques for the detection of ERPs in individual subjects for the purpose of clinical evaluations. Support vector machines (SVMs) are evaluated for their applicability as part of the protocol known as the Halifax Consciousness Scanner [19]. Our approach is hence mediating between a classical clinical approach and methods of BMIs.

The SVMs are a form of supervised statistical machine learning (for a primer, see [20]) and were first described by Vapnik and colleagues in 1992 [21]. When classified cases are used to train an SVM, it derives an optimal function based on the features that separate the two classes. Unlike other statistical classification methods, such as discriminant function analysis and linear regression, SVMs derive their decision function from only a subset of the data-the cases that are difficult to classify. These cases are referred to as support vectors. The goal of an SVM is to maximize the distance between the decision function's boundary and the support vectors. They can operate in either a linear or a nonlinear fashion and are notable among machine learning techniques in view of their ability to produce generalizable models from small datasets [22].

The SVMs have demonstrated very high accuracy for discriminating between experimental conditions [23], and moderately high accuracy for the prediction of clinical group membership [8] based on ERP features. The present work arose from the development of a novel method for evaluating brain function using ERPs, known as the Halifax Consciousness Scanner (HCS; described in [19]). The initial intent of the HCS is for applications in brain-injured populations. This context presents very different challenges from BMIs because patients may be unable to comply with task instructions [24]. It therefore uses stimulation parameters that do not require effortful task completion. This approach is also beneficial for clinical populations that may seek to influence their test results to conceal, augment, or even fake an injury [25]. Yet another important feature that distinguishes clinical applications from typical SVM applications in BMIs is the need for rapid ERP detection, without any kind of training period for the classifier. For this reason, we apply SVM training across subjects, rather than in the within-subject fashion that is typical for BMIs.

To evaluate the ability of an SVM to verify ERPs in individual subjects, the present study uses an ERP known as the mismatch negativity (MMN). First discovered by Näätänen and colleagues in 1978, the auditory MMN is an ERP that indexes the detection of change in a sequence of sounds [26]. The MMN is linked to a level of auditory memory that is believed to form the basis of conscious perception [27], and is an important part of the HCS method [19], particularly due to its well-established utility for coma monitoring [15, 28-32]. Owing to its small size, the MMN presents a challenge for detection at the individual subject level.

The MMN is commonly evoked using an experimental design known as an oddball paradigm. Although it is well understood that the oddball paradigm frequently causes an undesirable overlap of other ERPs with the MMN [33], it provides a simple framework for understanding how the MMN is obtained. In the oddball paradigm, a sequence of identical "standard" stimuli is interrupted at random and unpredictable times by "deviant" stimuli that possess some changed features, such as tonal frequency, intensity, or 
duration. The MMN is observed as an enhancement in negative voltage that occurs approximately $100-250 \mathrm{~ms}$ from deviant stimulus onset. It rests atop other ERP components that are associated with sensory processing, such as the "obligatory" $\mathrm{P} 1-\mathrm{N} 1-\mathrm{P} 2$ complex, and is isolated from these responses through a process of subtraction in which the averaged waveform to the standard stimuli is subtracted point-by-point from the averaged waveform to the deviant stimuli. This produces the so-called "difference wave," in which the MMN appears as a negative deflection in the 100-250 ms range. Although the amplitude of the MMN can be enhanced by experimental parameters such as reducing the silent period between stimuli (the interstimulus interval, or ISI), or increasing the difference between the standard and deviant tones [34], it is often quite small in amplitude, being around $1 \mu \mathrm{V}$.

The MMN is not restricted to simple oddball deviance and can also be evoked by more complex violations of concrete or abstract rules [35]. Under these circumstances, a pure MMN should be obtained, which is free of overlap from other ERP components. In the present study, the MMN is evoked in response to violations of a simple pattern of two alternating tones. Two types of SVM analyses are performed: one to achieve high classification accuracy $\left(\mathrm{SVM}_{\mathrm{acc}}\right)$, and the other to help one clarify whether the waveform features that are associated with the MMN are indeed used by the SVM $\left(\mathrm{SVM}_{\text {time }}\right)$. The SVM $\mathrm{Scc}_{\mathrm{acc}}$ analysis explores a variety of factors which could impact classifier accuracy: number of epochs entered into the averaged waveforms, scalp electrode selection, kernel type, and temporal window selection.

It is generally accepted that, as the number of epochs entered into the ERP waveform increases, the SNR of the ERP improves versus background EEG [1]. For the MMN, the number of epochs that is generally recommended for averaging is at least 150 [36], although robust MMNs have been observed with approximately 60 [34]. For this reason, the first SVM analysis explores the influence of number of epochs entered into the average on the accuracy of the classifier.

The typical scalp distribution for the MMN is maximal at frontal sites, and inverted at sites below the Sylvian fissure when a nose reference is used. This distribution has been attributed to bilateral, vertically oriented cortical generators on the supratemporal plane, as well as a frontal source [37]. Thus, a classifier that is limited to frontal sites might achieve the highest performance. However, for clinical populations such as patients with acquired brain injuries, atypical scalp distributions are expected [38]. For this reason, the value of using scalp electrode sites separately, or combined, is evaluated.

The so-called kernel trick permits SVMs to operate in a nonlinear fashion by projecting low-dimensional data into higher-dimensional space [20]. This may provide a better fit to complex datasets, but this improved accuracy may come at the cost of overfitting - a lack of generalizability of the results to new datasets [20]. For this reason, a linear classifier may be preferable, but the values of different kernel types are examined to determine whether significant improvements in performance can be achieved over the linear solution.

The MMN is expected to occur approximately $100-250 \mathrm{~ms}$ from stimulus onset [36]; however, other information in the ERP waveforms may be useful for classification. The portion of the waveform that is entered into the feature vectors is therefore varied, to determine what temporal window produces the maximum performance. A difficulty in the interpretation of SVMs, however, is that they derive their rules using a complex combination of factors. Any waveform features that are within the selected temporal window, be they part of the MMN or not, can contribute to the decision function. Thus, the $\mathrm{SVM}_{\text {time }}$ analysis is performed, not to achieve high accuracy, but to provide finer detail regarding which temporal regions of the waveforms contain detectable information for classification.

\section{Method}

\subsection{Subjects}

One hundred healthy adult volunteers (57 females, aged $19-73$, mean $=32.5$ years) with no history of neurological problems, hearing problems, or psychoactive medications participated in the study. The study was carried out according to the Canadian Tri-Council guidelines (Health, Natural, and Social Sciences) on ethical conduct for research involving humans.

\subsection{The stimulus sequence}

A segment of the tone sequence is illustrated in Fig. 1. This sequence was developed to evoke several ERPs for clinical applications such as brain injury [19]. It is composed of two spectrally rich tones (A and B) that consist of fundamental frequencies (740 and $1,046.5 \mathrm{~Hz}$, respectively) with the 1 st and the 2 nd harmonics presented at exponentially decreasing levels ( $\mathrm{e}^{-1}$ and $\mathrm{e}^{-2}$, respectively). These tones had durations of $100 \mathrm{~ms}$, including a 5-ms total rise/fall time, and were delivered with an onset-to-onset interstimulus interval of $265 \mathrm{~ms}$. The "standard" tones of the sequence were presented in an alternating pattern (ABABAB...) with an intensity of $75 \mathrm{~dB}$ SPL. Two types of deviant stimuli occurred infrequently within this standard pattern: repetition deviants (repetitions of either the A or the $\mathrm{B}$ tone, e.g., ABABBBA) and intensity deviants, both of which occurred with type A and type B fundamental 


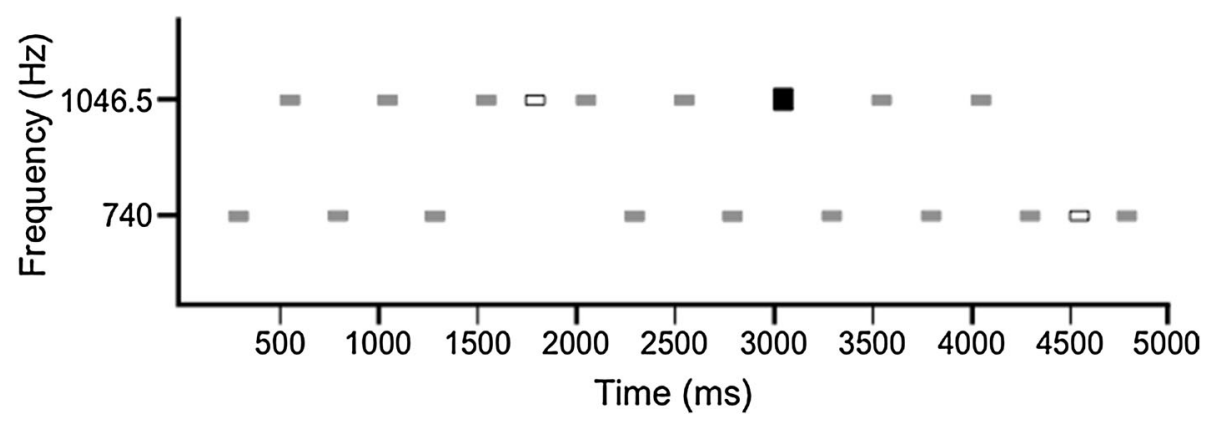

Fig. 1 An illustration of a segment of the 2.5-min tone sequence. Gray boxes are "standards" that conform to the pattern. Type A and type B tones have fundamental frequencies of 740 and $1,046.5 \mathrm{~Hz}$, respectively. The open box is a repetition deviant stimulus. The

frequencies. The sequential probabilities of repetition deviant and intensity deviant stimuli were 0.11 and 0.063 , respectively. Tones were delivered in a fixed order in which consecutive deviant stimuli (regardless of type) were separated by at least two standard tones. In total, the tone sequence consisted of 601 stimuli, consisting of 496 standard tones, 67 pattern deviants (e.g., ABABBBA), and 38 intensity deviants, and lasted for $2.5 \mathrm{~min}$.

\subsection{Data acquisition and analysis}

During sound presentation and EEG recording, subjects sat in a dimly lit room and watched a silent video with no subtitles. Subjects were asked to ignore all auditory stimulation and focus on the video. The EEG was recorded using a portable acquisition system consisting of a GmobiLab +8 channel amplifier (g.tec Medical Engineering, $\mathrm{GmbH}$ ), a custom-built triggering card, and a netbook. This configuration produced a small deflection in the EEG trace that was used to confirm the timing of stimulus delivery. Tin electrodes were placed at three scalp sites, including a Central site (at $\mathrm{Cz}$ ), a Frontal site $5.8 \mathrm{~cm}$ anterior to $\mathrm{Cz}$, and a Parietal site $5.5 \mathrm{~cm}$ posterior to $\mathrm{Cz}$. Assuming a distance of $35 \mathrm{~cm}$ between the nasion and inion, these Frontal and Parietal sites would lie approximately halfway between $\mathrm{Fz}$ and $\mathrm{FCz}$, and $\mathrm{Pz}$ and $\mathrm{CPz}$, respectively, according to the extended 10-20 system. Additional electrodes were placed at the right mastoid and on the tip of the nose. This configuration of a portable EEG system with a reduced electrode montage was optimized for point-of-care clinical applications [19]. The vertical and horizontal EOG were recorded from two electrodes positioned on the supraorbital ridge and outer canthus of the left eye, respectively. All sites were referenced to the left earlobe, and all impedances were below $5 \mathrm{k} \Omega$. The EEG and EOG signals were sampled at a rate of $256 \mathrm{~Hz}$, with a bandpass of $0.1-100 \mathrm{~Hz}$, and stored for offline analyses. thicker, black box, is an intensity deviant. Both repetition deviants and intensity deviants can have either a type A or type B fundamental frequency

Offline, these data were low-pass filtered at $20 \mathrm{~Hz}$ and notch filtered at $60 \mathrm{~Hz}$. Signals were re-referenced to the nose, and eye movement and blink artifacts were corrected using a regression method [39]. The continuous EEG was then segmented into discrete 1,000-ms epochs, including a 100-ms prestimulus period, and any epochs containing values exceeding $\pm 75 \mu \mathrm{V}$ were considered to contain artifacts and were rejected from further analysis. For the ERP analysis, all epochs were averaged, and baseline corrected using the $100-\mathrm{ms}$ prestimulus baseline. For greater detail regarding these standard ERP-processing steps, the interested reader is referred to [1]. In accordance with standard procedures for MMN analysis (e.g., [40]), the first four standard tones of the sequence, and the first 2-3 standards following every deviant were omitted from averaging. One participant's mastoid electrode became disconnected during the session; therefore the mastoid data from this subject were excluded from the grand average.

Averaging for the SVM analysis that explored the impact of the number of epochs on accuracy was performed using 2-40 epochs for each stimulus type. An upper limit of 40 epochs was selected as this was the lowest number of deviant epochs available per subject in the 100 -subject dataset. The data used for the SVM analysis did not receive baseline correction.

Similar to other research using two-tone alternating patterns [34, 40], standard and repetition deviant stimuli were averaged across tonal frequency stimulus types (e.g., all standard tones, regardless of whether they were low (A) or high (B) tones, were averaged together). The mean number of epochs accepted for averaging for each of these stimulus types was 214 for standard tone epochs and 66 for repetition deviant epochs. Data from the intensity deviant epochs, which evoke much larger ERPs and therefore do not test the sensitivity of the SVM approach, are not presented here. 


\subsection{ERP scoring}

The mean amplitude of the MMN was measured in a \pm 10 ms period surrounding its peak in each subject's repetition deviant minus standard difference wave. All amplitudes were measured versus baseline.

\subsection{Group level statistical analysis}

The MMN was tested for significance at the group level using a repeated-measures ANOVA with the factors electrode site (Frontal, Central, and Parietal) and stimulus type (repetition deviant and standard). Greenhouse-Geisser corrections were applied for all violations of the assumption of sphericity.

\subsection{SVM analysis for wave classification: $\mathrm{SVM}_{\mathrm{acc}}$}

\subsubsection{Pre-processing}

Feature vectors for SVM training consisted of two types of waveforms: separate ERPs for each scalp site, and a vector in which the data from the three scalp sites were appended in a Frontal to Parietal order (referred to as all sites). For the exploration of temporal window size, data from a prescribed temporal window within the averaged ERP waveform were used. These windows always began at stimulus onset ( $t=0 \mathrm{~ms}$ ), and varied from 20 to $900 \mathrm{~ms}$ in width in 20-ms steps. The features in each vector were EEG samples (sampled at $256 \mathrm{~Hz}$ ), therefore the number of features in each vector varied as a function of the size of the temporal window, and the number of electrode sites included in the vector. Illustrations of the types of feature vectors that were entered into the SVM analysis are provided in Fig. 2.

\subsubsection{SVM training}

Supervised learning was performed by 10 -fold cross-validation over 100 datasets (i.e., 100 subjects) to predict the fit of the model to a hypothetical validation set. In explicit terms, this means that in each fold, the SVM was trained on 90 subjects, and tested on 10 entirely different subjects that were held out.

First, to explore the value of the number of epochs that are entered into the average using a simple model, first, linear SVMs were trained with waveforms containing data from all sites. Different numbers of epochs were entered into the average (i.e., one standard tone epoch and one deviant tone epoch, or averaged standard and deviant waveforms consisting of $2,3, \ldots, 40$ epochs). In separate analyses, these epochs were either randomly selected from amongst the available set of epochs, or selected in serial order. As described in Sect. 2.3, the maximum number of epochs entered into these averages was 40 due to the fact that this was the lower limit of available deviant epochs within the 100 subject dataset.

Following the determination that SVM accuracy increased as the number of epochs entered into the average increased, averaged waveforms consisting of all available epochs were used as feature vectors to determine the influence of kernel type, temporal window size, and electrode selection. A series of SVM analyses were performed that included three factors: kernel type (linear, radial basis function $[\mathrm{RBF}]$, polynomial-cubic, and polynomial-quadratic), window size (between 100 and $900 \mathrm{~ms}$ in 20-ms steps, beginning at stimulus onset), and all electrode sites. All default values were retained for the different SVM kernels (e.g., linear SVM soft margin box constraint $C$ was left at 1 , and radial basis function scaling factor $\sigma=1$ was retained).

The final $\mathrm{SVM}_{\mathrm{acc}}$ solution that provided the best performance was finally evaluated using a standard permutation analysis, as described in the introduction of [41]. The analysis was carried out with 1,000 permutations. The SVM implementation used for this study was the built-in SVM toolbox in Matlab 2012b (MathWorks Inc., 2012). Autoscaling was enabled, which normalizes all input data vectors to have unit standard deviation and centers them around their mean.

2.7 SVM analysis to confirm MMN sensitivity: $\mathrm{SVM}_{\text {time }}$

\subsubsection{Pre-processing}

Averaged waveforms from all sites were used for the $\mathrm{SVM}_{\text {time }}$ analysis. Within this waveform, a 5 sample (approximately 20-ms) temporal window was defined, which slid by 5 sample steps across the entire 900 ms poststimulus epoch.

\subsubsection{SVM training}

Linear kernel SVMs were trained to distinguish between pattern deviant and standard tone averaged waveforms (all available epochs) using data from each 20-ms sliding window, to a total of 46 windows/SVMs. As in the $\mathrm{SVM}_{\mathrm{acc}}$ analysis, 10-fold cross validation was used across the 100 subjects. Again, the SVM implementation used for this study was the built-in SVM toolbox in Matlab 2012b (MathWorks Inc., 2012) with autoscaling enabled and the soft margin box constraint set to the default of 1 . 


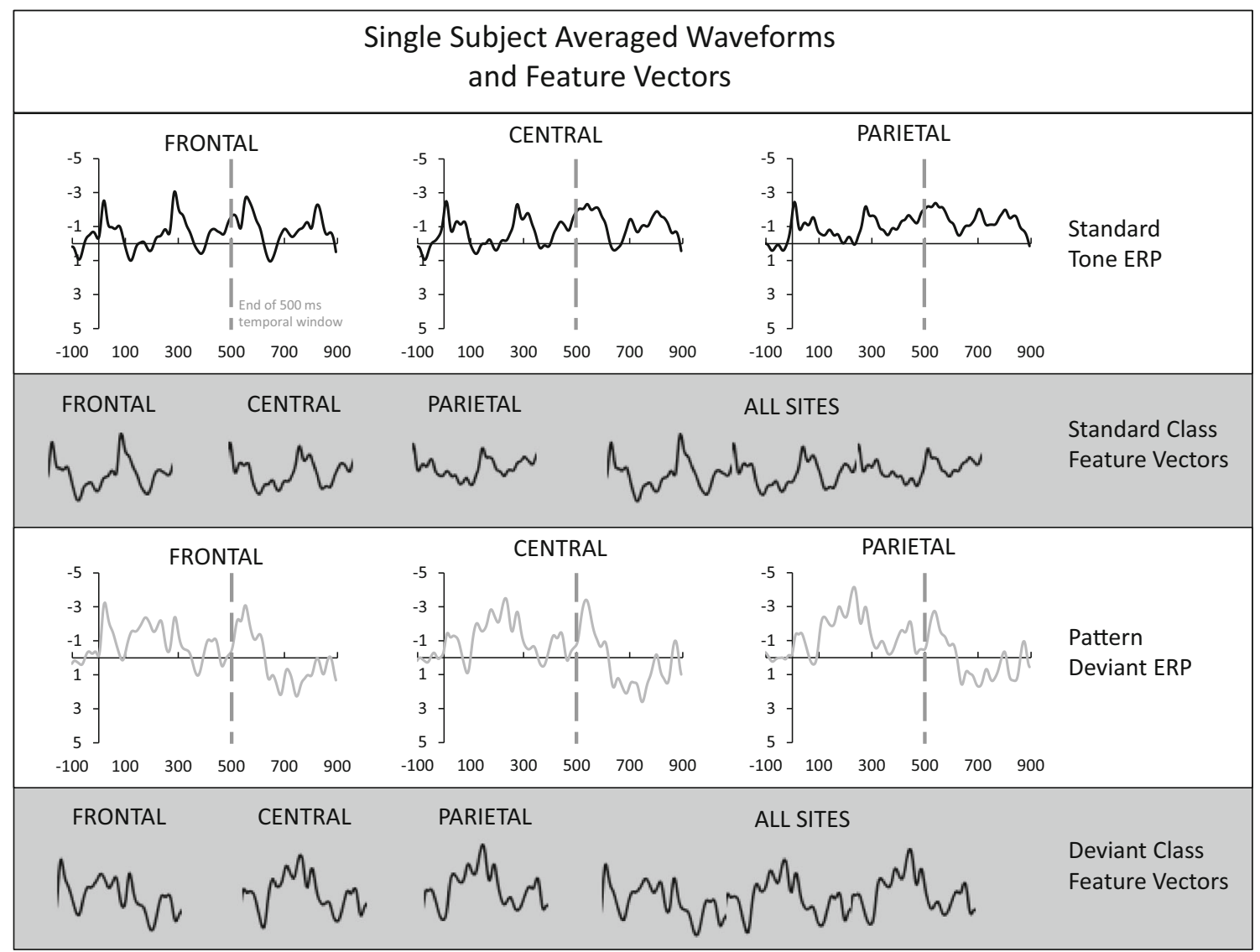

Fig. 2 An illustration of averaged waveforms and feature vectors for a single subject. The upper panel shows standard tone averaged waveforms at the three scalp sites (Frontal, Central, and Parietal) using all available epochs. All amplitudes are in microvolts and all time values are in milliseconds (horizontal axes). The end of a 500-ms temporal window is delimited by a gray dashed line. The middle upper panel demonstrates how the data from that temporal window

\section{Results}

\subsection{Group level ERP analysis}

Grand average waveforms for standard and deviant tones, as well as the deviant minus standard difference waves, are shown in Fig. 3. In the repetition deviant waveform, N1 was followed by an additional negativity that was maximal at the Frontal site and reversed in polarity at the mastoid. This is the MMN, which is isolated in the deviant minus standard difference wave. The average peak latency of the MMN was $193.0 \mathrm{~ms}$ from stimulus onset. The ANOVA for the MMN demonstrated a significant effect of stimulus type $\left[F(1,99)=97.7, p<0.001, \eta_{\text {partial }}^{2}=0.50\right]$ in which the mean amplitude at the latency of the MMN was more negative for repetition deviant than standard stimuli, thus, a significant MMN was evoked. There was also a significant effect of electrode site $[F(2,198)=21.2, \varepsilon=0.63$, adj. $\left.p<0.001, \eta_{\text {partial }}^{2}=0.18\right]$, in which amplitudes were more are used to create Frontal, Central, Parietal, and all scalp sites feature vectors for the standard ERP class. The lower middle panel shows pattern deviant averaged waveforms. Again, a 500-ms temporal window is delimited, and the composition of the Frontal, Central, Parietal, and all scalp sites feature vectors are shown, now for the deviant ERP class, in the bottom panel

negative at the Frontal site and the Central site than at the Parietal site. These main effects were qualified by an interaction $[F(2,198)=42.1, \varepsilon=0.70$, adj. $p<0.001$, $\left.\eta_{\text {partial }}^{2}=0.30\right]$ in which the MMN amplitude in the repetition deviant waveforms was larger at the Frontal site than the Parietal site, but not the Central site. The mean amplitude of the MMN, measured at the Frontal site in the deviant minus standard difference wave, was $-1.87 \mu \mathrm{V}$ (s.d. $=1.50 \mu \mathrm{V}$ ).

\section{2 $\mathrm{SVM}_{\mathrm{acc}}$ analysis}

As can be seen in Fig. 4, the accuracy of the linear SVM increased as a function of the number of epochs entered into the average using both randomly and serially selected epochs. However, accuracy rose much more sharply as a function of number of epochs for the serially than randomly selected condition. In a similar vein, the maximum accuracy that was achieved for serially selected epochs 
Grand Average

ERP Waveforms

FRONTAL
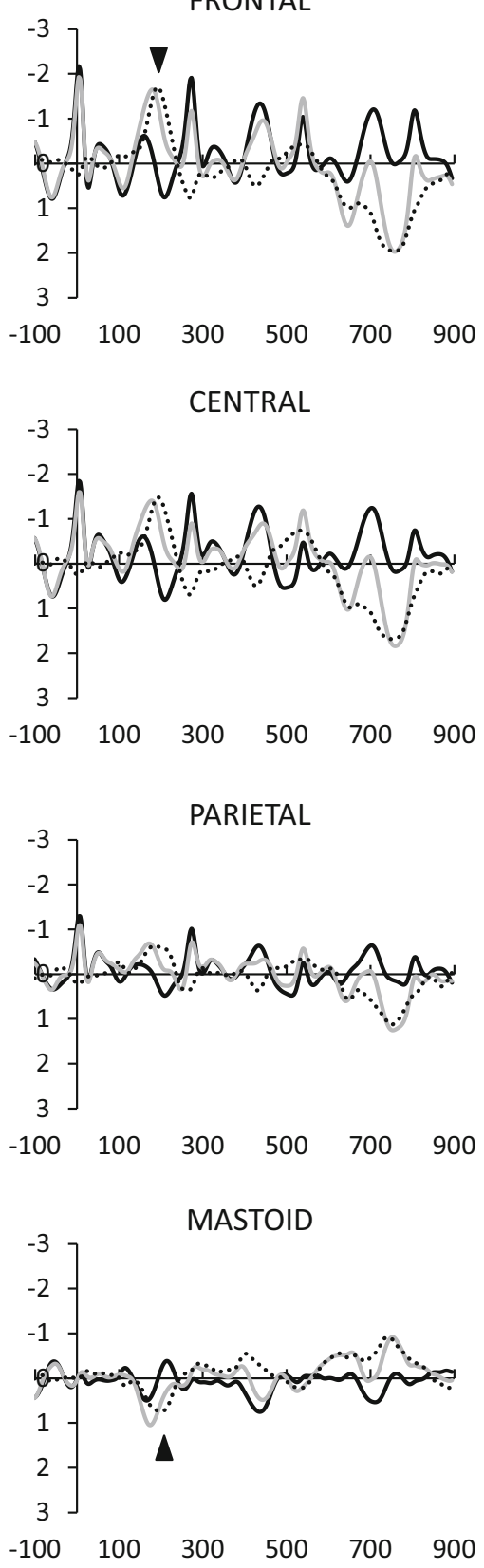

Fig. 3 Grand average waveforms for standard tones (black), repetition deviants (gray), and the deviant minus standard difference wave $($ dotted $)$. All amplitudes are in microvolts and all time values are in milliseconds. A sharp hardware-related deflection was observed at stimulus onsets (i.e., at $0 \mathrm{~ms}$ and every $265 \mathrm{~ms}$ thereafter). The MMN is indicated at the Frontal and Mastoid sites by an arrow. In the standard stimulus waveforms, obligatory auditory responses including $\mathrm{P} 1, \mathrm{~N} 1$, and P2 were apparent. Because of the rapid rate of stimulus delivery, these took on the appearance of a steady state response

$(78.13 \%, \mathrm{SD}=0.02 \%)$ was substantially higher than that which was obtained using randomly selected epochs $(65 \%, \mathrm{SD}=0.43 \%)$. Based on these results, further SVM analyses were performed on averages of all available epochs.

The results of varying kernel type, window size, and electrode selection are presented in Figs. 5 and 6. Figure 5 demonstrates the effect of kernel type and window size for a feature vector that contains all scalp electrodes. At window sizes below $180 \mathrm{~ms}$, the quadratic kernel provided maximum accuracy, and was significantly better than cubic or linear kernels. At a window size of $200 \mathrm{~ms}$, however, the linear classifier rose above all other classifier types, and performed significantly better than RBF or cubic classifiers. Unlike other kernel types, the accuracy of the linear SVM rose systematically as window size increased. Beginning with a 700-ms temporal window, the linear SVM provided significantly better performance than any other kernel type, and reached a maximum accuracy of $94.5 \%(\mathrm{SD}=0.064 \%)$ when the full post-stimulus epoch (900 ms) was used.

Figure 6 demonstrates the effect of electrode selection on a linear SVM, along with the effect of temporal window size. As in Fig. 5, it can be seen that classifier accuracy significantly improved when the temporal window reached at least $200 \mathrm{~ms}$ in width. Increasing the window width systematically improved classifier accuracy for SVMs trained at any site, but at the largest window size, the Frontal site, Central site, and the feature vector containing data from all sites produced significantly better performance than the Parietal site. Nearly equal performance was obtained for the Frontal site $(93 \%, \mathrm{SD}=0.048 \%)$, Central site $(92 \%, \mathrm{SD}=0.063 \%)$, and all sites $(94.5 \%$, $\mathrm{SD}=0.064 \%$ ).

The error of the final $\mathrm{SVM}_{\mathrm{acc}}$ solution (linear SVM, temporal window 0-900 ms, all sites) was evaluated using a permutation analysis [41], the results of which are shown in Fig. 7. The $p$ value for the accuracy of the true SVM solution was 0.001 , and was outside the $96 \%$ confidence interval of the accuracies that were observed with randomly assigned feature vectors.

\section{3 $\mathrm{SVM}_{\text {time }}$ analysis}

Accuracies within each of the nonoverlapping temporal windows are depicted in Fig. 8, and ranged from a minimum of $45.5 \%(\mathrm{SD}=0.09 \%)$ to a maximum of $86.5 \%$ $(\mathrm{SD}=0.01 \%)$. The highest accuracies were observed in the $160-260-\mathrm{ms}$ and $720-820-\mathrm{ms}$ periods.

\section{Discussion}

The tone sequence evoked a moderate-sized, frontally maximal MMN component that inverted in polarity at the mastoid site. This scalp distribution, though observed on a reduced array of sensors, conforms with the classic 
Fig. 4 Accuracy of a linear SVM as a function of the number of standard and deviant tone epochs entered into the average (using all scalp sites). Accuracy increased as the number of epochs entered into the average was increased from 2 to 40 , but increased much more steeply in the serial- than the random-selection condition, and reached a higher maximum when 40 epochs were used (78.13\%, SD $=0.02 \%$ for serial selection, versus $65 \%$, $\mathrm{SD}=0.043 \%$ for random selection). Error bars indicate standard deviation

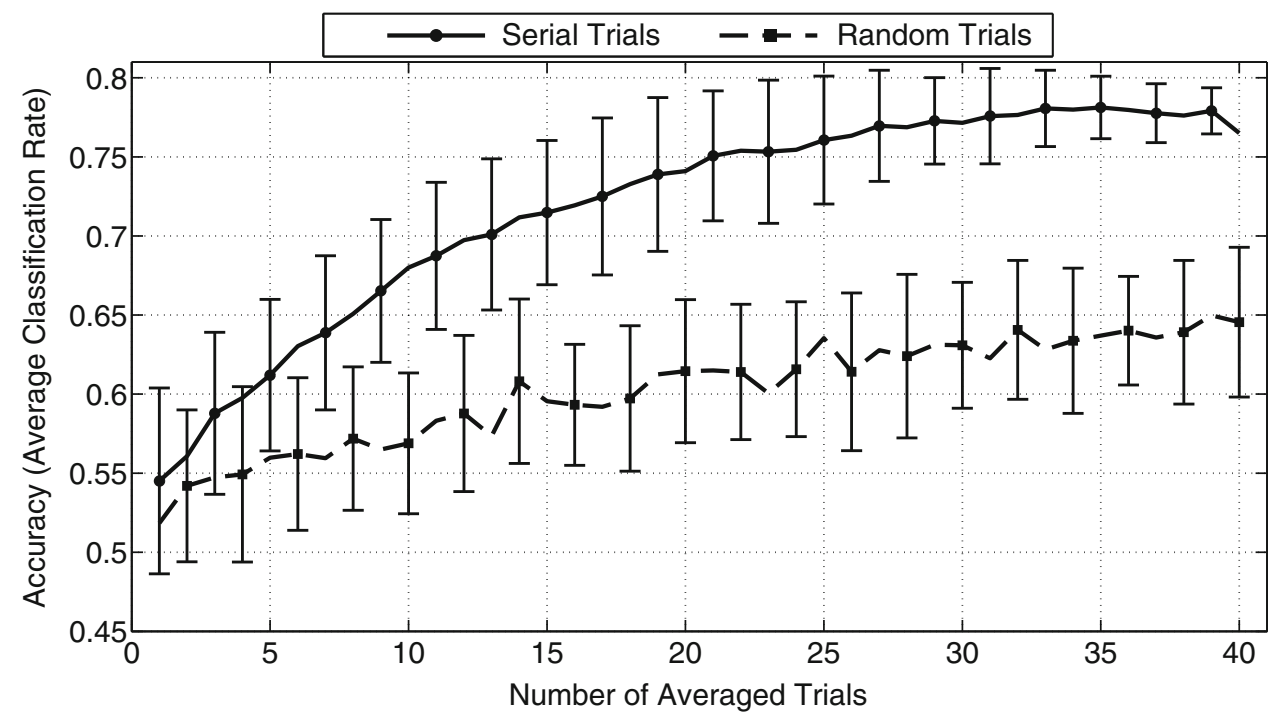

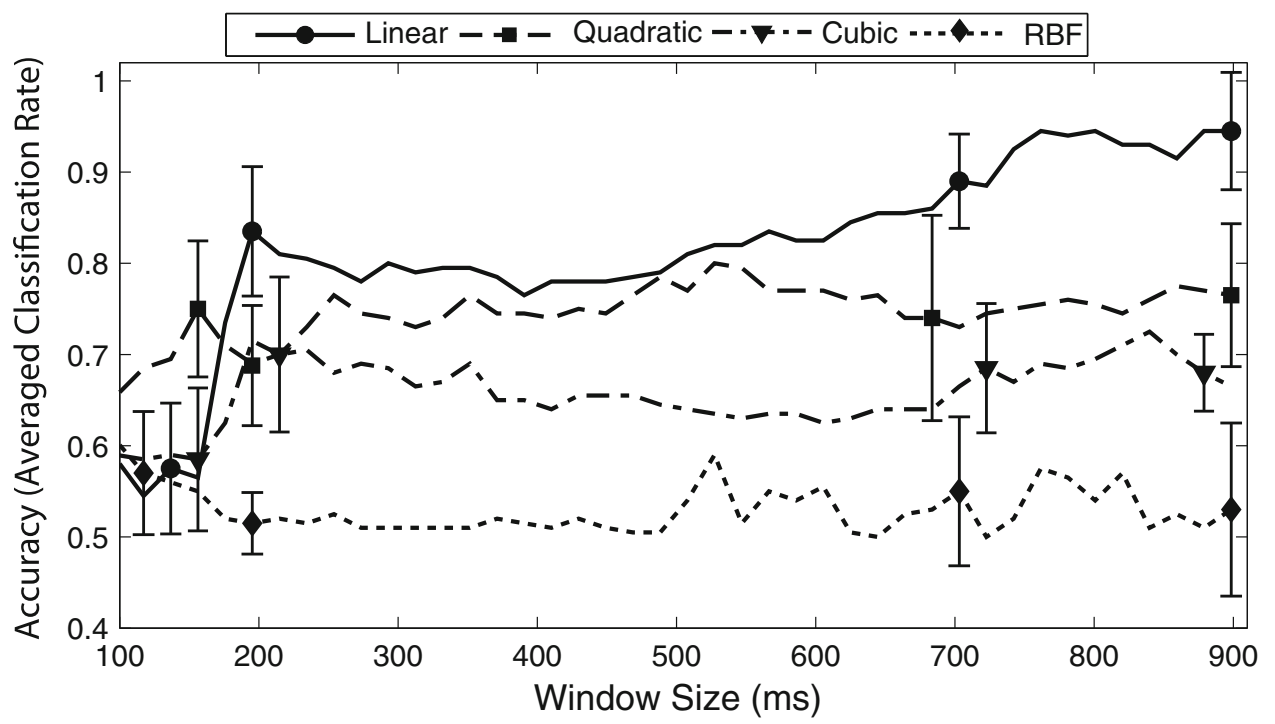

Fig. 5 Effects of SVM kernel type and temporal window size for a feature vector that contains all three scalp electrodes (average of all available epochs). At window sizes below $180 \mathrm{~ms}$, the quadratic kernel (dashed line with square) provided maximum accuracy, and was significantly better than other kernels. At a window size of $200 \mathrm{~ms}$, however, the linear classifier (solid line with circle) rose

morphology of the MMN and is identical to that observed using similar pattern paradigms [40]. The $\mathrm{SVM}_{\mathrm{acc}}$ analysis demonstrated that standard tone waveforms and deviant tone waveforms could be classified with $94.5 \%$ accuracy (as shown in Fig. 6 for the all sites feature vector at window size $900 \mathrm{~ms}$ ). These results bode well for the use of SVMs to detect ERPs for clinical applications, such as the ERP test battery approach that has been developed by our group, known as the Halifax Consciousness Scanner (described in [19], and a companion paper, [14]. above all other classifier types. Beginning at the 700-ms temporal window, the linear SVM provided significantly better performance than any other kernel type, and reached a maximum accuracy of $94.5 \%(\mathrm{SD}=0.064 \%)$ when the full post-stimulus epoch $(900 \mathrm{~ms})$ was used. For clarity, select error bars are displayed around 180, 200, 700, and 900-ms window sizes. Error bars indicate standard deviation

The performance of the SVM classifier was affected by a number of factors. First, the number of epochs used in the standard and deviant tone averaged waveforms was explored using a linear SVM. The accuracy of the classifier increased directly as a function of the number of epochs entered into the average. This result is entirely consistent with the general belief that the averaging process increases the SNRs of ERPs [1]. There was a consistent trend in which accuracy increased as the number of epochs entered into the average was increased from 2 to 40 . The effect of 


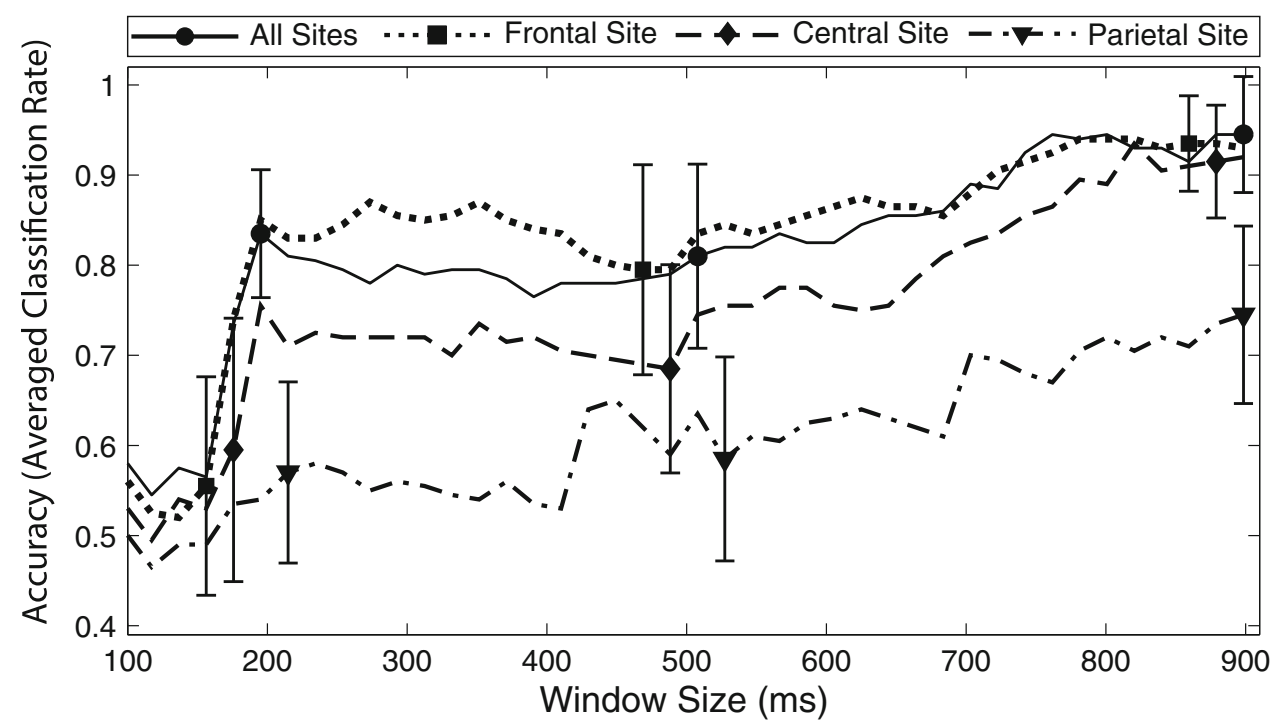

Fig. 6 Effects of electrode selection and temporal window size on a linear SVM trained with feature vectors that contain all three scalp electrodes (average of all available epochs). As in Fig. 5, increasing the window width systematically improved classifier accuracy. At the largest window size, the Frontal site (dotted line with diamond), Central site (dashed line with square), and the feature vector

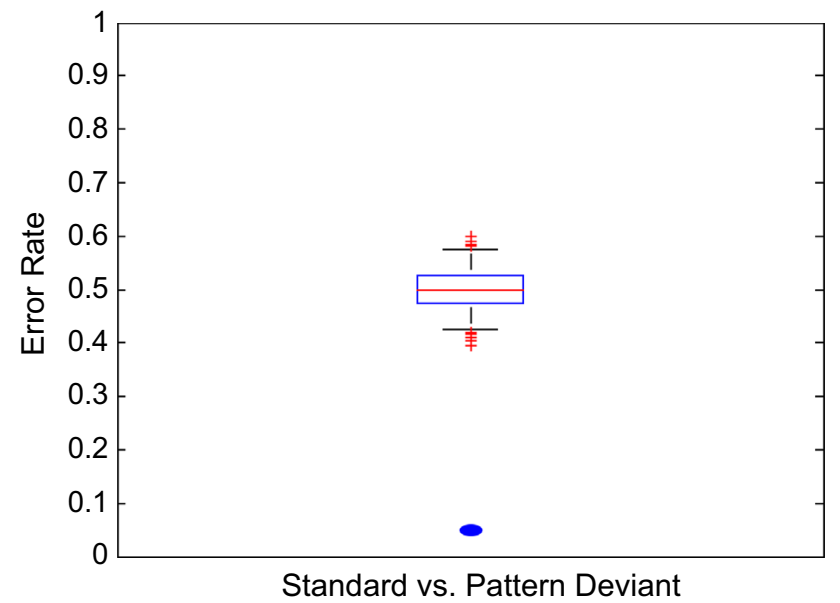

Fig. 7 Box and whisker plot for the null distribution produced by the permutation test for the final $\mathrm{SVM}_{\mathrm{acc}}$ solution (linear SVM, average of all available epochs, all sites, temporal window 0-900 ms). The center of the box is the median of the distribution. Box limits are the 25 th and 75 th percentiles, while the whiskers extend to the 2nd and 98th percentiles to delimit a two-tailed $4 \%$ threshold for significance. Accuracies that are observed in the null distribution, which exceed these limits are indicated by red cross marks. The observed accuracy of the true SVM solution (mean error $=5.5 \%$ ) is indicated by a blue ellipse. (Color figure online)

number of epochs on the accuracy of the SVM was much more dramatic for serially than randomly selected epochs, and achieved a higher accuracy when using 40 epochs in the serial condition. These results suggest that sequential effects that emerge over the stimulation protocol may have contributed to the accuracy of the classifier. containing data from all sites (solid line with circle) produced significantly better performance than the Parietal site (dash-dotted line with triangle). Nearly equal performance was obtained for the Frontal site, Central site, and all sites. For clarity, select error bars are displayed around 200, 500, and 900-ms window sizes. Error bars indicate standard deviation

The effect of averaging up to 67 epochs (the full available set) could not be explored across the group, due to the availability of less than 67 deviant epochs in some subjects as a result of artifact rejection. Nevertheless, the full set of available epochs was used for each subject in the final SVM solution, which achieved an accuracy of $94.5 \%$. Although the recommended number of epochs for MMN derivation is 150 , with a typical recording time of 6-12 min [36], the very high accuracy that was observed here with only 67 deviants, presented in an optimized 2.5-min sequence, is promising for clinical applications. While optimal recording conditions may be difficult to achieve, particularly in a clinical environment, the relatively small amount of data required for classification using this method represents a significant reduction in testing time versus conventional stimulation parameters. Short testing times are necessary in a clinical context because they interfere minimally with routine care procedures and avoid fluctuations in vigilance, attention, and fatigue which can cause false negatives on ERP-based tests [42].

Other factors that influenced SVM accuracy were the type of kernel (linear, RBF, cubic, or quadratic), the temporal window used, and the electrode site used. When all kernel types were explored across different temporal windows, using a feature vector with data from all sites, the linear SVM clearly outperformed other kernel types. This effect was particularly noticeable as the temporal window size approached $200 \mathrm{~ms}$ - the period in which the MMN begins to emerge. At the maximum window size, $900 \mathrm{~ms}$, 
Fig. 8 Accuracies of 46 linear SVMs trained using data in nonoverlapping 20-ms sliding temporal windows across the entire $900-\mathrm{ms}$ post-stimulus period of the averaged waveform (all available epochs, all sites). The highest accuracies were achieved in the 160-260 and 720-820 ms periods (maximum 86.5 and $83.5 \%$, respectively)

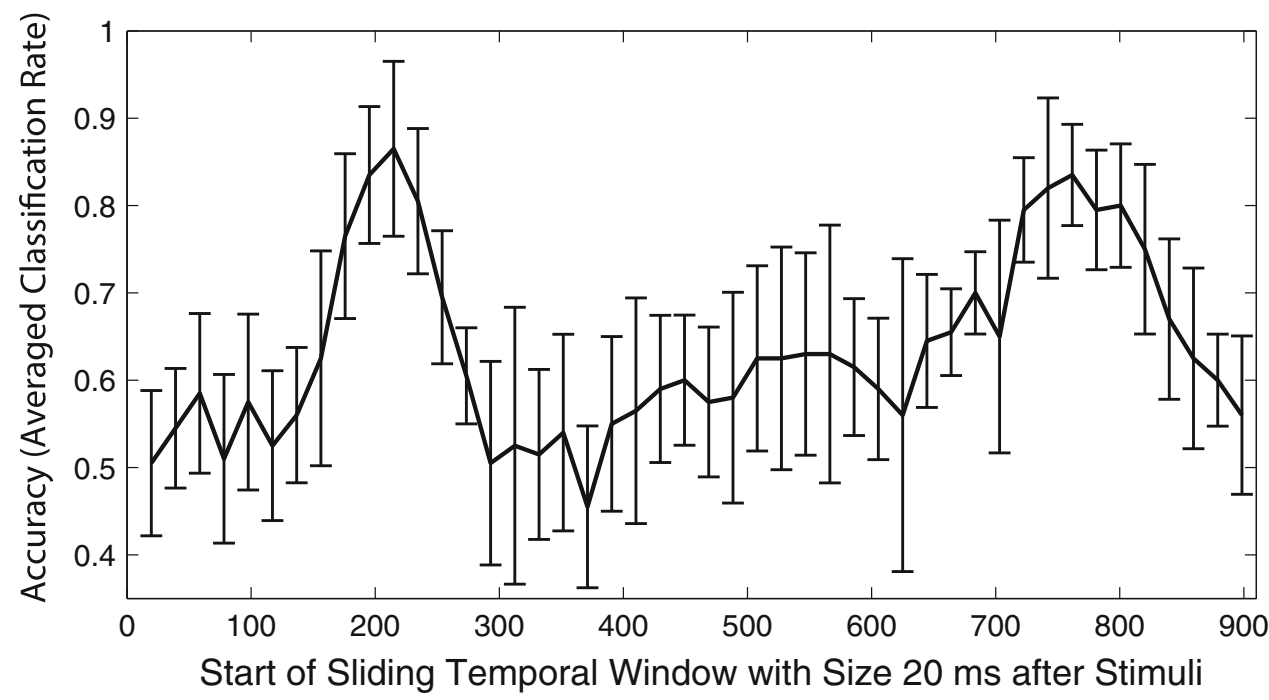

the linear SVM performed significantly better than all other kernel types, particularly the RBF, which never rose above $59 \%$. It remains possible that optimizing the parameters of each SVM kernel type could improve performance for the nonlinear classifiers. More complex kernel types, however, can lead to overfitting, and a lack of generalizability [20]. For this reason, the effect of parameter optimization was not explored for the nonlinear classifiers.

The electrode site that was entered into the feature vector also had a significant impact on the accuracy of the classifier. In line with the known scalp distribution of the MMN [37], feature vectors from the Frontal and Central site produced significantly better results than ones from the Parietal site. Similarly, feature vectors containing data from all three scalp sites produced very high performance, which was not significantly different from the classifiers that were trained exclusively with data from the Frontal and Central sites. This approach may be preferable in clinical populations whose scalp distributions can vary from that typically observed in healthy individuals.

For the linear SVM, increasing the width of the temporal window entered into the feature vector had a direct impact on the accuracy of the classifier, in which increasing the width of the window systematically improved performance. The most dramatic improvement in accuracy occurred around $200 \mathrm{~ms}$, near the peak of the MMN in the grand average (193 ms). Maximum accuracy was observed when the full post-stimulus epoch was utilized (900 ms).

One drawback of SVMs for classification, as opposed to other statistical classifiers like discriminant function analysis, is that their decision functions use complex combinations of information that can make their interpretation difficult. Thus, it is unknown which ERPs or brain oscillations that lie in the employed temporal window are contributing to the performance of the classifier.
To gain insight into the waveform features that contribute most to classification accuracy, a secondary analysis $\left(\mathrm{SVM}_{\text {time }}\right)$ was performed. Of the $46 \mathrm{SVMs}$ trained in nonoverlapping 20-ms (5 sample) windows, those in the 160-260 and 720-820 ms range achieved the highest accuracy (first period maximum $=86.5 \%, \mathrm{SD}=0.1 \%$, second period maximum $=83.5 \%, \mathrm{SD}=0.058 \%)$. The first period corresponds well with the expected latency of the MMN to the pattern deviant, which peaked on average at $193 \mathrm{~ms}$. The second period corresponds with a region of positivity in the deviant tone response, which could reflect a P3-type response. While both $\mathrm{MMN}$ and $\mathrm{P} 3$ indicate that deviance detection has occurred, P3 is linked to subsequent cognitive operations like attention switching and working memory updating [43]. Regardless of the true component structure of this positive response, it is clear that a classifier which uses this portion of the waveform does not detect the MMN per se, but rather uses a larger variety of waveform features to perform the task of classifying waveforms associated with deviance detection.

In conclusion, linear SVMs proved to be effective at classifying the MMN. This method could be extremely valuable for clinical applications of the MMN, and other ERPs, by both reducing testing time versus conventional stimulation parameters and permitting the application of ERPs at the single subject level. Among the potential applications for the MMN is monitoring the status of severely brain-injured patients $[15,19,28,32]$. Further development for use in this patient population may require additional testing to determine efficacy in the context of the severely altered EEG patterns that are commonly observed in this group [38]. Results must also be interpreted with care when applied in the absence of more concrete indicators of neurological status [44-47]. 
Acknowledgments Primary development of the HCS was funded by the National Research Council of Canada (NRC), Canada's Natural Sciences and Engineering Research Council of Canada (NSERC), and the Province of Nova Scotia (Innovacorp). While being employees of the Government of Canada, Drs. Petley and D'Arcy, were also inventors on the initial patent describing the intellectual property for the HCS, which is wholly owned by the Crown (international PCT application number CA2011050367). Their inventorship may qualify them to receive a small percentage of royalties from the Crown. The HCS is under exclusive license to Mindful Scientific, Inc., for which Dr. D'Arcy now serves as Chief Scientific Advisor. The work described herein was funded by NSERC through an Engage grant to Dr. Trappenberg for industry-partnered research with Mindful Scientific, Inc.

Open Access This article is distributed under the terms of the Creative Commons Attribution License which permits any use, distribution, and reproduction in any medium, provided the original author(s) and the source are credited.

\section{References}

1. Luck SJ (2005) An introduction to the event-related potential technique. MIT Press, Cambridge

2. Näätänen R, Picton T (1987) The N1 wave of the human electric and magnetic response to sound: a review and an analysis of the component structure. Psychophysiology 24(4):375-425

3. Hood LJ (1998) Clinical applications of the auditory brainstem response. Singular Publishing, San Diego

4. Stapells DR (2009) Cortical event-related potentials to auditory stimuli. Handbook of clinical audiology. Lippincott Williams \& Wilkins, New York, pp 395-430

5. Connolly JF, D'Arcy RC (2000) Innovations in neuropsychological assessment using event-related brain potentials. Int $\mathrm{J}$ Psychophysiol 37(1):31-47

6. Connolly JF, D’Arcy RC, Lynn Newman R, Kemps R (2000) The application of cognitive event-related brain potentials (ERPs) in language-impaired individuals: review and case studies. Int $\mathbf{J}$ Psychophysiol 38(1):55-70

7. Gawryluk JR, D'Arcy RC, Connolly JF, Weaver DF (2010) Improving the clinical assessment of consciousness with advances in electrophysiological and neuroimaging techniques. BMC Neurol 10:11

8. Stahl D, Pickles A, Elsabbagh M, Johnson MH (2012) Novel machine learning methods for ERP analysis: a validation from research on infants at risk for autism. Dev Neuropsychol 37(3):274-298

9. Bostanov V, Kotchoubey B (2006) The t-CWT: a new ERP detection and quantification method based on the continuous wavelet transform and Student's t-statistics. Clin Neurophysiol 117(12):2627-2644

10. Hu L, Mouraux A, Hu Y, Iannetti GD (2010) A novel approach for enhancing the signal-to-noise ratio and detecting automatically event-related potentials (ERPs) in single trials. Neuroimage 50(1):99-111

11. Zou L, Zhang Y, Yang LT, Zhou R (2010) Single-trial evoked potentials study by combining wavelet denoising and principal component analysis methods. J Clin Neurophysiol 27(1):17-24

12. Iyer D, Zouridakis G (2007) Single-trial evoked potential estimation: comparison between independent component analysis and wavelet denoising. Clin Neurophysiol 118(3):495-504
13. Ponton CW, Don M, Eggermont JJ, Kwong B (1997) Integrated mismatch negativity (MMNi): a noise-free representation of evoked responses allowing single-point distribution-free statistical tests. Electroencephalogr Clin Neurophysiol 104(2):143-150

14. Sculthorpe-Petley L, Parvar H, Ghosh Hajra S et al. A rapid event-related potential (ERP) method for point-of-care evaluation of brain function: Development of the Halifax Consciousness Scanner. Under Review

15. Morlet D, Bouchet P, Fischer C (2000) Mismatch negativity and N100 monitoring: potential clinical value and methodological advances. Audiol Neurootol 5(3-4):198-206

16. D'Arcy RC, Marchand Y, Eskes GA et al (2003) Electrophysiological assessment of language function following stroke. Clin Neurophysiol 114(4):662-672

17. Marchand Y, D'Arcy RC, Connolly JF (2002) Linking neurophysiological and neuropsychological measures for aphasia assessment. Clin Neurophysiol 113(11):1715-1722

18. Mirghasemi H, Fazel-Rezai R, Shamsollahi MB (2006) Analysis of p300 classifiers in brain computer interface speller. Conf Proc IEEE Eng Med Biol Soc 1:6205-6208

19. D'Arcy RC, Hajra SG, Liu C, Sculthorpe LD, Weaver DF (2011) Towards brain first-aid: a diagnostic device for conscious awareness. IEEE Trans Biomed Eng 58(3):750-754

20. Noble WS (2006) What is a support vector machine? Nat Biotechnol 24(12):1565-1567

21. Boser BE, Guyon I, Vapnik V (1992) A training algorithm for optimal margin classifiers. In: Proceedings of the fifth annual workshop of computational learning theory, 5. ACM, Pittsburgh pp $144-152$

22. Vapnik V (1999) The nature of statistical learning theory. Springer, New York

23. Merzagora AC, Butti M, Polikar R et al (2009) Model comparison for automatic characterization and classification of average ERPs using visual oddball paradigm. Clin Neurophysiol 120(2):264-274

24. Wang JT, Young GB, Connolly JF (2004) Prognostic value of evoked responses and event-related brain potentials in coma. Can J Neurol Sci 31(4):438-450

25. Jeter CB, Hergenroeder GW, Hylin MJ, Redell JB, Moore AN, Dash PK (2013) Biomarkers for the diagnosis and prognosis of mild traumatic brain injury/concussion. J Neurotrauma 30(8):657-670

26. Näätänen R, Gaillard AW, Mantysalo S (1978) Early selectiveattention effect on evoked potential reinterpreted. Acta Psychol (Amst) 42(4):313-329

27. Näätänen R, Winkler I (1999) The concept of auditory stimulus representation in cognitive neuroscience. Psychol Bull 125(6):826-859

28. Fischer C, Morlet D, Bouchet P, Luaute J, Jourdan C, Salord F (1999) Mismatch negativity and late auditory evoked potentials in comatose patients. Clin Neurophysiol 110(9):1601-1610

29. Fischer C, Morlet D, Giard M (2000) Mismatch negativity and N100 in comatose patients. Audiol Neurootol 5(3-4):192-197

30. Fischer C, Luaute J, Adeleine P, Morlet D (2004) Predictive value of sensory and cognitive evoked potentials for awakening from coma. Neurology 63(4):669-673

31. Fischer C, Luaute J, Nemoz C, Morlet D, Kirkorian G, Mauguiere $\mathrm{F}$ (2006) Improved prediction of awakening or nonawakening from severe anoxic coma using tree-based classification analysis. Crit Care Med 34(5):1520-1524

32. Daltrozzo J, Wioland N, Mutschler V, Kotchoubey B (2007) Predicting coma and other low responsive patients outcome using 
event-related brain potentials: a meta-analysis. Clin Neurophysiol 118(3):606-614

33. Jacobsen T, Schröger E (2001) Is there pre-attentive memorybased comparison of pitch? Psychophysiology 38(4):723-727

34. Alain C, Woods DL, Ogawa KH (1994) Brain indices of automatic pattern processing. NeuroReport 6(1):140-144

35. Picton TW, Alain C, Otten L, Ritter W, Achim A (2000) Mismatch negativity: different water in the same river. Audiol Neurootol 5(3-4):111-139

36. Duncan CC, Barry RJ, Connolly JF et al (2009) Event-related potentials in clinical research: guidelines for eliciting, recording, and quantifying mismatch negativity, P300, and N400. Clin Neurophysiol 120(11):1883-1908

37. Giard MH, Perrin F, Pernier J, Bouchet P (1990) Brain generators implicated in the processing of auditory stimulus deviance: a topographic event-related potential study. Psychophysiology 27(6):627-640

38. Kotchoubey B, Lang S, Mezger G et al (2005) Information processing in severe disorders of consciousness: vegetative state and minimally conscious state. Clin Neurophysiol 116(10):2441-2453

39. Gratton G, Coles MG, Donchin E (1983) A new method for offline removal of ocular artifact. Electroencephalogr Clin Neurophysiol 55(4):468-484

40. Sculthorpe LD, Collin CA, Campbell KB (2008) The influence of strongly focused visual attention on the detection of change in an auditory pattern. Brain Res 1234:78-86

41. Ojala M, Garriga GC (2010) Permutation tests for studying classifier performance. J Mach Learn Res 11:1833-1863

42. Neumann N, Kotchoubey B (2004) Assessment of cognitive functions in severely paralysed and severely brain-damaged patients: neuropsychological and electrophysiological methods. Brain Res Brain Res Protoc 14(1):25-36

43. Polich J (2007) Updating P300: an integrative theory of P3a and P3b. Clin Neurophysiol 118(10):2128-2148

44. Cruse D, Chennu S, Chatelle C et al (2011) Bedside detection of awareness in the vegetative state: a cohort study. Lancet 378(9809):2088-2094

45. Goldfine AM, Victor JD, Conte MM, Bardin JC, Schiff ND (2012) Bedside detection of awareness in the vegetative state. Lancet 379(9827):1701-2; author reply 1702

46. Goldfine AM, Bardin JC, Noirhomme Q, Fins JJ, Schiff ND, Victor JD (2013) Reanalysis of "Bedside detection of awareness in the vegetative state: a cohort study". Lancet 381(9863):289-291

47. Cruse D, Chennu S, Chatelle C et al (2013) Reanalysis of "Bedside detection of awareness in the vegetative state: a cohort study"—authors' reply. Lancet 381(9863):291-292

Hossein Parvar received his B.Sc. degree in computer hardware engineering from Iran University of Science and Technology (IUST) in 1998, and his M.Sc. and Ph.D. degrees in computer architecture engineering from the Science and Research branch of Islamic Azad University, Iran in 2001 and 2012, respectively. He is now adjunct professor at the Faculty of Computer Science, Dalhousie University. His research interests include machine learning, hierarchical intelligent systems architecture, and distributed decision-making systems.

Lauren Sculthorpe-Petley received her Ph.D. in Psychology from the University of Ottawa in Ottawa, Canada, in 2010. She is currently a research scientist with the Biomedical Translational Imaging Centre (BIOTIC), located in the QEII Health Sciences Centre and the IWK Health Centre in Halifax, Canada. She is also an Assistant Professor with Dalhousie University. Her expertise is in auditory event-related potentials, and, more recently, MEG. Her research focuses on developing novel diagnostics for hearing deficits and brain injury.

Jason Satel received his B.Sc. in Psychology and Biology from Saint Mary's University (Canada), and his Masters and Ph.D. degrees in Computer Science from Dalhousie University (Canada). He is currently an Assistant Professor in the School of Psychology at the University of Nottingham Malaysia Campus. His research interests are in Cognitive Science, primarily Visual Attention, using multiple interdisciplinary techniques (Neuroimaging, Psychophysics, and Computational Modeling).

Rober Boshra received a bachelor of science in Computer Science with honors first class from Dalhousie University in 2014. Currently a $\mathrm{PhD}$ student in the neuroscience program (MiNDS) at McMaster University, he is working on formulating directed neural networks in the human brain and their implication in speech cognition using machine learning techniques. His research interests include automatic feature selection and detection, sparse representations of neuroimaging data, unsupervised machine learning, and brain-computer interfacing.

Ryan C.N. D'Arcy received his B.Sc. (with distinction) from the University of Victoria (Canada), and then his M.Sc. and Ph.D. in Neuroscience from Dalhousie University (Canada). He did his postdoctoral training in MRI physics at the National Research Council Canada, Institute for Biodiagnostics. He is a Professor in Engineering Science and Computer Science at the Simon Fraser University and holds the Surrey Memorial Hospital Foundation BC Leadership Chair in Multimodal Technology for Healthcare Innovation. He is also Head of Health Sciences and Innovation at Surrey Memorial Hospital and the Fraser Health Authority. His research specializes in brain-imaging technologies and translating medical research to real-world benefits for patient care.

Thomas P. Trappenberg received a PhD in Physics from Aachen University, Germany, and held research positions at Dalhousie University, Canada, the RIKEN Brain Science Institute, Japan, and Oxford University, England. He is a full professor in Computer Science and author of Fundamentals of Computational Neuroscience, now in its second edition. His research interests are computational neuroscience, machine learning, and neurocognitive robotics. 